\title{
Le coton en Afrique de l'Ouest et du Centre: de la révolution agricole à la transition agro-écologique
}

\author{
Mamy Soumaré ${ }^{1, *}$, Michel Havard ${ }^{2,4, \dagger}$ et Bruno Bachelier ${ }^{3,4}$ \\ ${ }^{1}$ Institut d'Economie Rurale, Université des Sciences Sociales et de Gestion, Bamako, Mali \\ 2 CIRAD, UMR Innovation, IER, Bamako, Mali \\ ${ }^{3}$ CIRAD, UPR AIDA, F-34398 Montpellier, France \\ ${ }^{4}$ Univ Montpellier, Montpellier, France
}

\begin{abstract}
Résumé - Depuis les années 1970, le coton a été un moteur dans la transformation des systèmes de production agricole et un élément structurant des économies locales dans les savanes d'Afrique de l'Ouest et du Centre (AOC). Cependant, la durabilité des systèmes de production à base de coton est souvent mise en débat à cause de leurs effets sur les sols et des impacts négatifs de la lutte chimique sur l'environnement et la santé humaine. Au moyen de réformes drastiques, le secteur coton en AOC a surmonté les crises, montrant sa capacité de résilience. Aujourd'hui, ce secteur est confronté à des défis importants sur lesquels la recherche doit se mobiliser: (i) économiques (volatilité des prix, revenus des producteurs, bien-être des communautés), (ii) sociaux (inclusion des couches vulnérables, rôle des femmes et des jeunes, etc.) et (iii) environnementaux (santé humaine, impact des pesticides, dégradation des sols, etc.) et liés au changement climatique. Ce numéro thématique présente des résultats et perspectives de travaux de recherche pouvant contribuer à améliorer la durabilité des systèmes cotonniers en AOC, à ouvrir des pistes vers une transition agro-écologique et à faire face aux nouveaux défis.
\end{abstract}

Mots clés : Coton / développement rural / agro-écologie / durabilité / Afrique de l'Ouest et du Centre

\begin{abstract}
Cotton in West and Central Africa: from the agricultural revolution to the agro-ecological transition. Since the 1970s, cotton has been a driving force in the transformation of agricultural production systems and a structuring element of local economies in the savannah areas of West and Central Africa (WCA). However, the sustainability of cotton based production systems is often debated because of their effects on soils and the negative impacts of chemical pest control on the environment and human health. Through drastic reforms, the WCA cotton sector overcame the crises, showing its resilience. Today, this sector is facing major challenges on which research must be mobilized: (i) economic (price volatility, producers' income), (ii) social (inclusion of vulnerable groups, role of women and young people, etc.) and (iii) environmental (impact of pesticides, soil degradation, etc.) and linked to climate change. The articles published in this issue present some results and perspectives of research work that can contribute to improve the sustainability of cotton systems in WCA, to open paths towards an agro-ecological transition, and to address the new challenges.
\end{abstract}

Keywords: Cotton / rural development / agro-ecology / sustainability / West and Central Africa

\section{Introduction}

On parle de révolution agricole si, dans un système agraire donné, les exploitations agricoles progressent et adoptent de nouveaux moyens de production, développent de nouvelles

\footnotetext{
*Auteur de correspondance : soumare_mamy@hotmail.com

†Auteur décédé.
}

pratiques et de nouveaux systèmes de culture et d'élevage et créent un nouvel écosystème cultivé pour faire émerger un nouveau système agraire (Mazoyer et Roudard, 2000). Les effets induits par le coton dans les savanes de l'Afrique de l'Ouest et du Centre francophone (AOC) ont fait évoluer les systèmes agraires traditionnels à culture entrecoupée de jachère vers de nouveaux systèmes agraires avec des espaces cultivés en continu grâce à l'intensification des pratiques et en particulier l'utilisation d'intrants. Le secteur mondial du coton 


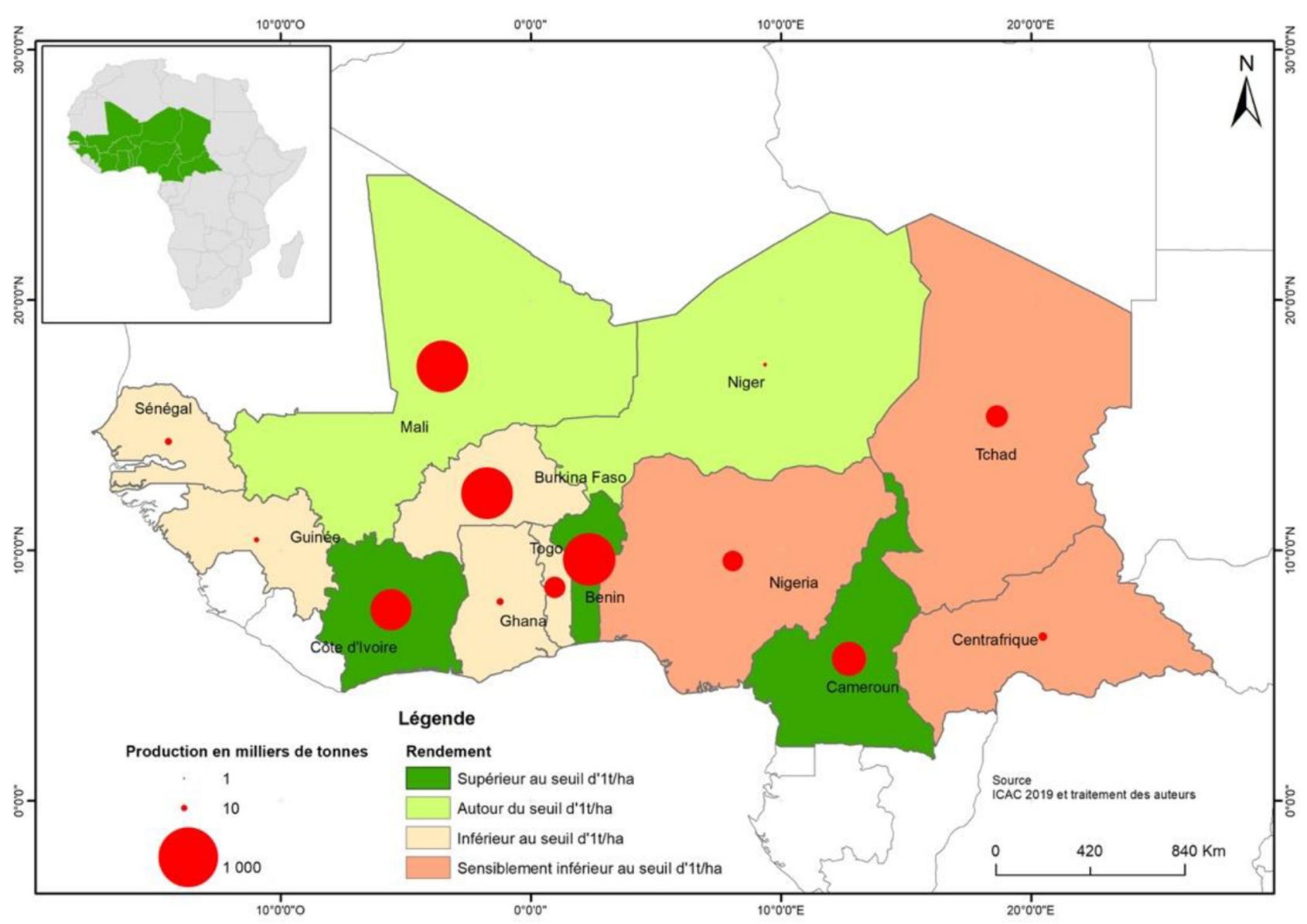

Fig. 1. Production et rendement en coton graine en Afrique de l'Ouest et du Centre.

Fig. 1. Production and yield of seed cotton in West and Central Africa.

englobe plus de 100 millions d'exploitations familiales réparties dans 75 pays mais, pour beaucoup de ces exploitations, le coton n'est qu'un des éléments d'un système de production plus complexe et intégré (FAO, 2015).

En AOC, les premiers essais de la culture du coton ont été réalisés au Sénégal dans les années 1820 (Barry, 2014), et la culture commença à s'y développer après la première guerre mondiale (Levrat, 2008; p. 46). Aujourd'hui, les pays cotonniers africains de la zone Franc CFA constituent l'une des grandes zones de production de coton à travers le monde. Selon le Comité Consultatif International du Coton (CCIC ou International Cotton Advisory Committee-ICAC), ils participent à hauteur de 12 à $15 \%$ aux exportations mondiales de fibres (ICAC, 2019). En 2018-2019, ces zones ont fourni 4,5\% des 26 millions de tonnes de fibre de coton mondiale soit près de 3,3 millions de tonnes de coton graine (ICAC, 2019). Dans les pays producteurs d'AOC, le coton contribue pour $3 \%$ à $15 \%$ au produit intérieur brut (PIB) (Planetoscope, 2018). Mais la production reste concentrée au Bénin, au Burkina Faso, au Cameroun, en Côte d'Ivoire et au Mali. En 2019, la production annuelle se situait entre 300000 et 750000 tonnes de coton graine dans chacun de ces pays, qui sont aussi ceux qui ont les meilleurs rendements en culture pluviale, avec des valeurs supérieures à une tonne à l'hectare (ICAC, 2019) (Fig. 1).

\section{La culture du coton : élément structurant des économies agricoles des pays producteurs en AOC}

La dynamique commencée sous la colonisation s'est amplifiée durant la période post-Indépendance, au cours de laquelle la culture du coton a été au cœur des politiques agricoles et du développement de plusieurs pays d'AOC. Elle est progressivement devenue un élément structurant des systèmes de production agricole et des économies locales. Ainsi, le bassin cotonnier africain de la zone Franc CFA a vu sa part dans la production de coton subsaharienne passer de $13,5 \%$ en 1970 à environ $65 \%$ en 2018-2019 (ICAC, 2019).

La fameuse citation de René Dumont (1962) «l'Afrique noire est mal partie » a eu un écho important et continue d'en avoir. À propos du coton, Dumont disait au moment des Indépendances : "l'introduction de cultures de traite (coton, arachide) n'a pas été un moyen de modernisation des méthodes agricoles; au contraire, elle a appauvri les terres $\gg$. Cependant, la progression rapide de la production de fibres de coton et ses effets sur le développement rural en AOC entre 1961 (48 000 tonnes) et 2004 (1 127000 tonnes) (ICAC, 2019), en font une réussite rare en Afrique subsaharienne, comme le montrent les qualificatifs illustrant 
les transformations induites par cette culture: "Révolution agricole», "Success story», "Développement inégalé», «Filière intégrée favorisant la diffusion du progrès technique et de l'innovation», "Cercles vertueux du progrès» (PeltreWurtz et Steck, 1991 ; Bassett, 2002 ; Devèze et des Fontaines, 2005 ; Renaudin, 2010).

Cependant, des analyses plus critiques de la filière cotonnière pointent les impacts environnementaux négatifs de la culture et les rémunérations modestes qu'en tirent les agricultures familiales (Pichot et al., 2006), la faible proportion du coton transformé dans la sous-région, la volatilité et la tendance baissière des prix du coton, la distorsion occasionnée par les subventions des États-Unis et des pays européens (Nubukpo et Keita, 2006), ou encore l'intoxication des producteurs par les pesticides (Westerberg et al., 2017). La crise sécuritaire des années 2010 touche principalement les zones sahéliennes et les productions de céréales; elle affecte peu les zones cotonnières d'AOC, excepté les fortes baisses de production de coton au Tchad et en République Centrafricaine : la situation est cependant aussi fragile au Nord du Cameroun et à l'Est du Burkina Faso (Commodafrica, 2019; Le Point Afrique, 2019).

\section{Le coton: moteur du développement rural des régions cotonnières d'AOC}

En AOC, la culture du coton a pu se maintenir sur les mêmes territoires durant des décennies, et devenir la locomotive des systèmes de culture grâce : (i) à l'engagement des exploitations agricoles familiales bénéficiant de l'accès aux intrants et au crédit de campagne, (ii) à l'accompagnement efficace des services techniques, de la recherche, des organisations de producteurs (OP), mais également (iii) à des politiques publiques volontaristes et des partenaires financiers, en particulier, à partir des années 1970, la Caisse centrale de coopération économique (CCCE) (devenue Caisse française de développement-CFD) en 1989, puis Agence française de développement - AFD - en 2001).

Mis en place au cours des années 1970, le modèle d'organisation basée sur une intégration verticale de la filière a été presque le même dans tous les pays d'AOC. Une compagnie cotonnière nationale a pris la place de la Compagnie française de développement des textiles (CFDT) avec un capital majoritairement détenu par l'État et une participation de la CFDT: Société de développement du coton du Cameroun (Sodécoton), Société burkinabè des fibres textiles (Sofitex), CotonTchad, etc. En plus de l'encadrement de la production, de l'égrenage et de la commercialisation de la fibre de coton, ces sociétés nationales avaient des missions de développement rural qui leur avaient été assignées par l'État : (i) construction de pistes rurales nécessaires à l'évacuation du coton, (ii) alphabétisation des producteurs facilitant la gestion des associations de producteurs, (iii) appui aux productions animales par la valorisation des tourteaux de coton et la traction animale, accompagnement des autres cultures entrant en rotation avec le coton (maïs, mil, sorgho) ou concourant à l'équilibre alimentaire des exploitations (riz). Elles s'appuyaient sur des organisations de producteurs (comme l'Association villageoise au Mali) dont le rôle allait au-delà de la sphère du coton: construction de salles de classe et de centres de santé, lotissement des villages, recrutement des enseignants et des agents de santé, etc. Cet extrait de Soumaré (2008) illustre cette dynamique au Mali : "Dans le village de Siramana (à $30 \mathrm{~km}$ de Sikasso), on a assisté à un déplacement $d u$ site du village; le premier site (vieux village ou village traditionnel) a été définitivement abandonné au profit d'un nouveau site où de nouvelles maisons sont construites avec des formes géométriques, des toits en tôles et des rues bien droites. Les habitants racontent avec fierté ce changement qui s'est produit avec l'argent du coton $\gg$.

Il en a résulté des transformations importantes: développement de la culture attelée, intensification des systèmes de culture grâce à l'utilisation de la fumure organique et des engrais minéraux, intégration agriculture-élevage rendue possible par l'investissement des revenus monétaires du coton dans l'élevage et émergence d'une élite et d'un mouvement paysan influents sur les scènes politiques.

\section{Le cas du Mali, une illustration du succès du coton}

$\mathrm{Au}$ plan technique, le nombre des exploitations équipées d'un attelage complet (une paire de bœufs de trait, une charrue, un multiculteur équipé de corps sarcleur et butteur) est passé de 10 à $95 \%$ de 1970 à 2000 . Le nombre de bœufs de trait a été multiplié par six, passant de 100000 à 600000 en 30 ans (Fig. 2). La production en coton a entraîné le développement des cultures céréalières comme le maïs, dont la production est passée de 60000 à plus de 700000 tonnes de 1975 à 2018 .

Alors qu'on aurait pu s'attendre en parallèle à une forte utilisation des pesticides, celle-ci est restée relativement faible grâce à l'évolution des méthodes de lutte (lutte étagée ciblée-LEC, traitement sur seuil) ayant permis la maîtrise de la pression parasitaire (Michel et al., 2000). Grâce à l'adaptation des méthodes de lutte phytosanitaire, le nombre de traitements insecticides est resté limité à 3 ou 4 , même après 50 ans de culture (Castella et Deguine, 2006).

$\mathrm{Au}$ plan économique, c'est au secteur cotonnier que la Banque nationale pour le développement agricole (BNDA) et l'institution de microfinance Kafo Jiginew (grenier de la communauté) doivent leur développement, avec la gestion des crédits de campagne et des crédits pour les équipements agricoles confiés par la Compagnie malienne de développement des textiles (CMDT) (Ouédraogo et Gentil, 2008). Au plan social et politique, bon nombre des élus communaux et des maires sont d'anciens secrétaires et responsables des associations de producteurs de coton. Certains ont même réussi à briguer des mandats nationaux à l'Assemblée nationale et au Haut conseil des collectivités territoriales.

\section{L'essoufflement et les réformes du secteur cotonnier en AOC}

Après 30 années de croissance continue, le secteur cotonnier d'AOC a commencé à présenter des signes d'essoufflement à partir des années 2000. Cette période difficile d'une dizaine d'années, qualifiée de crise cotonnière, était le résultat des fluctuations et de la baisse tendancielle des cours internationaux de la fibre, de la faillite économique des États et du reflux des aides à l'agriculture (Gafsi et Mbetid-Bessane, 2003), mais aussi 


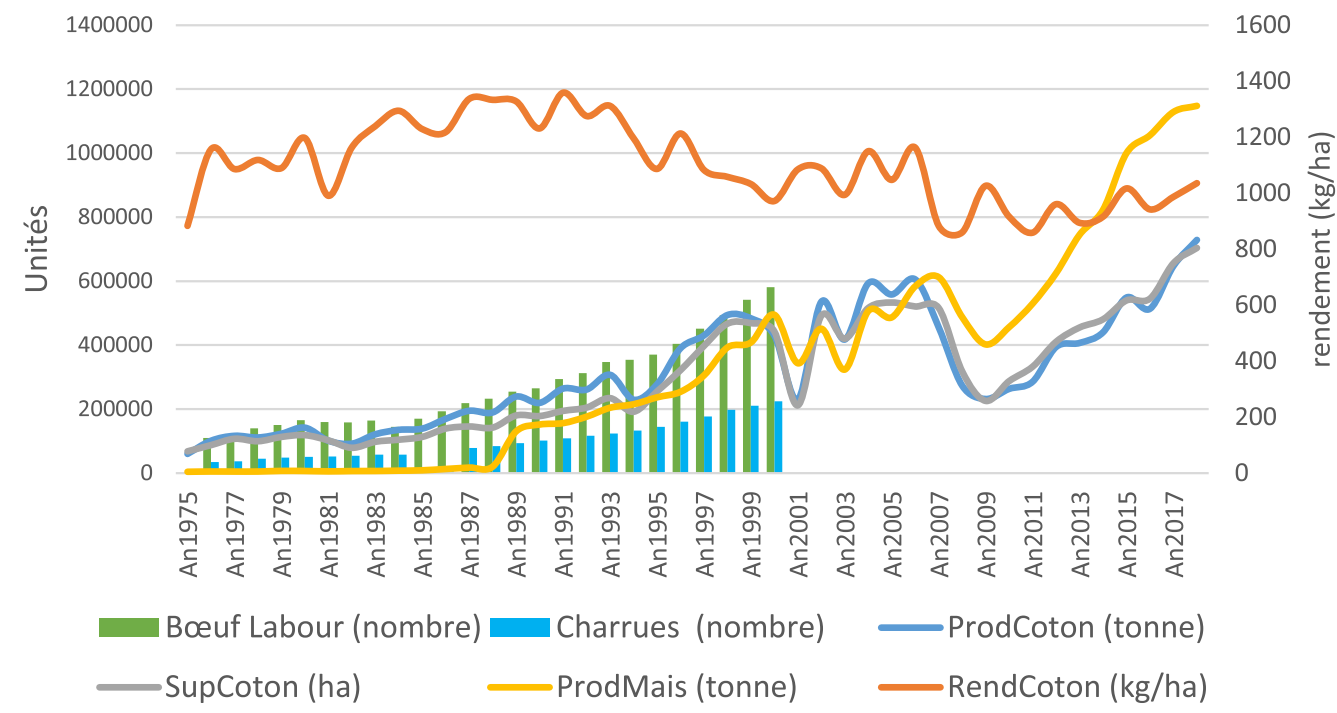

Source : CMDT

Fig. 2. Évolution des productions, des superficies, des rendements en coton et maïs et du niveau d'équipement des exploitations. Fig. 2. Evolution of production, areas, cotton and corn yields and level of farm equipment.

de facteurs structurels (dysfonctionnement des filières, erreurs de gestion) dans les sociétés cotonnières (Mbétid-Bessane et al., 2010). Cette crise s'est manifestée par des prix faibles aux producteurs de coton, la baisse des surfaces cultivées, voire celle des rendements, entraînant la baisse des productions (près de $50 \%$ entre 2005 et 2008) (Fig. 2), le boycott de la culture du coton par les paysans comme au Mali, la désorganisation des systèmes de production et des systèmes de cultures liée aux difficultés d'accès aux intrants et au crédit, l'insécurité alimentaire (Folefack et al., 2014). Elle s'est aussi traduite par des déficits importants des sociétés cotonnières liés à la baisse des cours mondiaux du coton et au maintien de prix relativement élevés au producteur, ainsi que par le désarroi dans les zones rurales africaines. "Cette crise est particulièrement grave, dans la mesure où elle engage le devenir de millions de personnes dont les revenus dépendent directement ou indirectement de cette production $\gg$ (Renaudin, 2010).

Face à cette crise, les solutions proposées furent nombreuses et souvent contradictoires. Deux grandes théories s'affrontèrent. À la vision financière des institutions de Bretton Woods (Banque mondiale et Fonds monétaire internationalFMI), plaidant pour la privatisation et le recentrage des sociétés cotonnières sur le coton, s'opposait une vision, dite intégrée, s'appuyant sur l'argumentaire suivant : la question du coton dans cette zone n'est pas que financière, elle est surtout économique et sociale. Cette posture adoptée par l'Agence française de développement (AFD) était partagée par beaucoup de pays (par exemple le Burkina Faso et le Mali, voir le cas de ce dernier en Encadré 1). Par contre, au Bénin, la filière avait été libéralisée depuis les années 1990. Elle ne faisait plus intervenir une société cotonnière unique, mais plusieurs acteurs : des égreneurs, des unions de producteurs et les distributeurs d'intrants (Nubukpo, 2011). Ce changement n'a pourtant pas permis de résoudre les difficultés de la filière (baisse des cours du coton, hausse du prix des intrants, etc.), et l'État a été amené en 2007-2008 à apporter une subvention pour soutenir le prix au producteur (Nubukpo, 2011).
Encadré 1. Déclaration du Président malien Amadou Toumani Touré sur les réformes du secteur cotonnier

Ces déclarations du Président malien Amadou Toumani Touré, citées par Orsenna (2006), attestent bien l'embarras des gouvernants d'alors: «En cinq ans la pauvreté a reculé de $5 \%$ dans les zones cotonnières et augmenté de $2 \%$ ailleurs. Aujourd'hui l'or blanc est en train de devenir notre malédiction. Le coton fait vivre près $d u$ tiers de notre population: trois millions et demi d'hommes et de femmes! Comment voulez-vous que nous renoncions au coton. C'est vrai,j'ai accepté de garantir au paysan un prix supérieur au cours mondial. Comment pouvais-je faire autrement? Ils se soulevaient! C'est ça la volonté de la Banque mondiale: une autre zone d'instabilité, dans le sud de notre pays, aux frontières de la Côte d'Ivoire d'où ne cessent d'arriver des réfugiés? Comment voulez-vous que je les nourrisse? On nous accable pour notre déficit, mais personne n'aborde les causes de ce déficit. Depuis 40 ans, jour après jour, nous avons lutté pour nous améliorer. Nous avons joué à fond le jeu de la concurrence sans la moindre chance de gagner puisque le plus puissant triche. Et contre la guerre des monnaies entre l'Europe et les États-Unis que pouvonsnous? Par notre appartenance à l'euro, nos pieds et poings sont liés puisque le coton est acheté en dollar. Vous trouvez ça normal? Un pays parmi les plus pauvres accroché à la monnaie la plus haute. Plus elle grimpe plus nous tombons. Personne ne proteste et surtout pas la Banque mondiale». Ces déclarations ont conduit la Banque mondiale et le FMI à suspendre leur appui au gouvernement du Mali en 2006. Quelques années plus tard, quand la Banque mondiale a fait le choix de soutenir l'agriculture, le Président malien ironisait en ces termes « Je suis content que la Banque m'ait retrouvé sur ce chemin ». 
Même si tous n'ont pas suivi la voie de la libéralisation totale comme le Bénin, tous les pays producteurs de coton se sont engagés dans des processus de réformes drastiques: recentrage des sociétés cotonnières sur la production et l'égrenage du coton, restructuration des sociétés en plusieurs sociétés ou filiales avec réduction des effectifs de personnels et création d'interprofessions du coton.

Au Burkina Faso, l'État a ouvert le capital de la Sofitex aux producteurs à travers l'Union nationale des producteurs de coton (UNPC-B). En 2004, la Sofitex a dû céder deux zones de production, représentant $20 \%$ de la production nationale, à deux nouvelles sociétés, Faso Coton et la Société cotonnière du Gourma (Socoma) (Zoundi et al., 2006; FMI, 2014).

Au Mali, la CMDT a été divisée en quatre zones de production, structurées chacune en une filiale dans l'optique d'une mise en vente. Mais les premiers appels d'offres ont été infructueux. L'État a donc continué à soutenir la CMDT et les producteurs de coton pendant des années, pour un coût de plusieurs milliards de Francs CFA. La Société Dagris (exCFDT et actuel Géocoton) a cédé ses actions à l'État du Mali pour se contenter de $0,51 \%$ du capital. L'État est alors devenu actionnaire principal avec 99,49\%. Il céda ensuite $20 \%$ des capitaux de chacune des quatre filiales à la Confédération des sociétés-coopératives des producteurs de coton (C-SCPC).

Les leçons d'une étude de la Banque mondiale (Tschirley et al., 2009a ; 2009b) sur les réformes de l'organisation et les performances des filières cotonnières africaines mettent en évidence qu'une concurrence accrue n'améliore pas forcément les performances des filières et que le rôle que doivent jouer les pouvoirs publics dépend des tendances du marché international et $\mathrm{du}$ dispositif de régulation interne du secteur cotonnier.

\section{Résilience et défis du secteur cotonnier en Afrique de l'Ouest et du Centre}

Dans l'ensemble, le secteur cotonnier a pu faire face à ces difficultés, et montrer sa résilience malgré l'ampleur de la crise des années 2000. Depuis une dizaine d'années, les pays de la zone Franc CFA sont redevenus des acteurs du marché mondial. Certains ont battu ou égalé leur record de production annuelle: 743000 tonnes de coton-graine au Mali en 20172018 (PR-PICA, 2018), 710000 tonnes au Burkina Faso en 2014-2015 (Commodafrica, 2015 ; RFI, 2015) et 720000 tonnes au Bénin en 2018-2019 (Afcot, 2019).

Mais ces pays ont des difficultés à maintenir leur niveau de production et les revenus de la culture du coton sur le long terme. Ainsi, la production du Burkina Faso est passée de près de 700000 tonnes en 2006 à 400000 tonnes en 2009 (Lankoandé et al., 2011). Depuis les années 2000, les rendements en coton-graine stagnent et tournent autour de 1 tonne à l'hectare. Les revenus de la culture du coton, malgré leur importance relative, peinent à satisfaire les besoins monétaires de certaines catégories d'exploitations, qui finissent par devoir recourir à des prêts à des taux usuriers, atteignant $100 \%$ de taux d'intérêt (Sossou et Fok, 2019, dans ce numéro thématique).

La durabilité des systèmes de cultures cotonniers en AOC est régulièrement mise en cause au vu de leurs impacts potentiels négatifs sur l'environnement et la santé humaine.
À titre d'exemple, à l'Ouest du Burkina Faso, plus de $80 \%$ des sols des systèmes de culture cotonniers du Mouhoun sont dégradés (dégradation du substrat avec pertes en terres et appauvrissement chimique) (Hauchart, 2008). Au Bénin, de nombreux producteurs sont intoxiqués chaque année par les pesticides utilisés sur le coton (Westerberg et al., 2017). Lors du colloque Beating Famine, tenu du 26 au 28 février 2019 à Bamako, une session organisée par la GIZ (Gesellschaft für Internationale Zusammenarbeit, coopération allemande) était intitulée "Le coton en Afrique de l'Ouest: or blanc ou cause de la destruction des sols et des dommages environnementaux ». Si certaines de ces postures sont questionnables au plan scientifique, car s'appuyant plus sur des études locales à caractère de généralisation limité, la question de l'avenir de la «success story» de la culture du coton se pose cependant eu égard aux enjeux contemporains de l'agriculture.

En effet, en dépit de la résilience du secteur cotonnier africain, des défis anciens demeurent et de nouveaux apparaissent: (i) la volatilité du prix de la fibre sur le marché mondial, (ii) l'amélioration et la sécurisation du revenu des producteurs et du bien-être des communautés rurales, (iii) la modification des équilibres biologiques et de la répartition des bio-agresseurs, (iv) la réduction des impacts négatifs des systèmes de culture à base de coton sur l'environnement par l'amélioration de la gestion de la fertilité des sols et de la gestion du paysage et par la réduction de l'utilisation des pesticides, (v) les interférences politiques dans la gestion de la filière coton. D'autres défis importants ne sont pas spécifiques des systèmes à base de coton et sont à relever par l'agriculture en général: (i) le développement de pratiques agricoles plus écologiques et adaptées aux nouvelles contraintes imposées par le changement climatique, (ii) l'inclusion des couches vulnérables de la population dans la dynamique de développement agricole, particulièrement les femmes et les jeunes, (iii) le maintien des jeunes dans les exploitations agricoles.

En Afrique, selon le Groupe d'experts intergouvernemental sur l'évolution du climat (IPCC, 2014), le changement climatique entraînera des perturbations de la température, de la teneur en $\mathrm{CO}_{2}$ atmosphérique et des précipitations, avec des impacts sur les cultures (dont le coton), notamment en termes de rendement, de précocité et de résistance aux ravageurs et aux maladies. Pour s'adapter à ces perturbations et atténuer leurs impacts négatifs, la mise en œuvre de systèmes de culture à base de coton durables, modifiés ou innovants, doit contribuer à la conservation des ressources et de la biodiversité. Pour ce faire, plusieurs voies sont possibles (Bachelier et al., 2018) : (i) la gestion durable des terres, en mobilisant les processus d'intensification écologique des systèmes de culture et de production, (ii) la gestion de la matière organique du sol, avec des effets sur la rétention d'eau, l'érosion ou la disponibilité des nutriments, (iii) la gestion agro-écologique des ravageurs et des maladies, (iv) la sélection de variétés précoces, compactes et résistantes aux effets de perturbations environnementales.

Dans les exploitations de petite taille en AOC, une grande partie du travail de production est assurée par les femmes, depuis le semis des cultures jusqu'à la récolte (FAO, 2015). Ce rôle est rarement reconnu ou récompensé (ITC, 2011). Dans la filière cotonnière, contrairement aux hommes, les femmes ont difficilement accès aux crédits, sont peu représentées dans l'organisation collective et encourent des risques sanitaires liés à l'utilisation de pesticides (Schneider et al., 2010). 
Ce sont ces défis qui nous ont amenés à faire un point sur la façon dont ils sont relevés - ou pas - dans un certain nombre de pays ouest-africains. Tous les défis ne sont cependant pas abordés dans les articles de ce numéro thématique, qui fait suite à celui réalisé en 2006 (Cahiers Agricultures, 2006, Numéro spécial: Le coton, des futurs à construire - https:// revues.cirad.fr/index.php/cahiers-agricultures/issue/view/ 3297).

\section{Vers des systèmes cotonniers plus durables?}

Des initiatives sont en cours au niveau international pour évaluer l'effet des pratiques agricoles et de leurs évolutions sur la durabilité de la culture cotonnière et pour amorcer une dynamique vers des systèmes de culture et d'élevage plus écologiques. Le panel SEEP (Social, Environmental and Economic Performance of cotton), créé en 2006 sous l'égide de l'ICAC, a élaboré une liste d'indicateurs pour mesurer la durabilité des systèmes de culture du coton (FAO, 2015). Celle-ci prend en compte les défis liés à la gestion des ravageurs, de l'eau et des sols, à la biodiversité et à l'utilisation des terres, au changement climatique, à l'équité et au genre, au travail des enfants. Par ailleurs, la Better Cotton Initiative $(B C I)$ (https://bettercotton.org/about-bci/), les projets d'appui à la transition agro-écologique aux échelles régionales et nationales (Bénin, Burkina Faso et Mali) et la création de départements en charge de l'environnement au sein des sociétés cotonnières montrent la nécessité et la volonté de transformation des systèmes de production actuels vers des systèmes plus écologiques: réduction de l'utilisation des intrants chimiques, promotion de l'agro-écologie et de la culture biologique, diversification et utilisation des produits biologiques en culture conventionnelle.

Les articles publiés dans ce numéro thématique des Cahiers Agricultures concernent les progrès réalisés en matière d'agriculture, de sécurité alimentaire et d'accès à l'éducation et à la santé. Ils visent à discuter l'amélioration de la durabilité des conditions de production dans les systèmes cotonniers en AOC et ouvrent des pistes vers une transition agro-écologique de cette agriculture.

Une bonne partie des articles traitent des pratiques de gestion des ravageurs dans les systèmes de production, afin de les rendre plus économes en pesticides, plus respectueux de la santé humaine et de l'environnement, et plus résilients. Un accent particulier est mis sur l'approfondissement des connaissances du système de vie des ravageurs et de leurs ennemis naturels (Brévault et al., 2019), pour travailler sur des approches visant à stimuler les processus écologiques de régulation naturelle des ravageurs (Brévault et al., 2019) et à réduire l'utilisation des pesticides : coton Bt (Vognan et Fok, 2019) ou écimage du cotonnier (Diarra et al., 2020). Ensuite, il est important d'informer, de sensibiliser et de former les acteurs de la filière sur les dangers liés à l'utilisation des pesticides sur la santé et sur l'environnement (Djagani et Fok, 2019) et sur les bonnes pratiques à adopter pour la lutte chimique, depuis le choix des pesticides jusqu'à leur application au champ (Le Bars et al., 2020). Ces travaux sur la gestion des ravageurs du cotonnier montrent que des résultats ont été obtenus sur la réduction de l'utilisation des pesticides en culture cotonnière, comme au Togo entre 1990 et 2010 (Djagani et Fok, 2019), alors que, dans le même temps, le plan Ecophyto lancé en France avec les mêmes objectifs s'est révélé être un échec (Guichard et al., 2017). Une autre voie de contrôle des ravageurs est l'utilisation d'organismes génétiquement modifiés (OGM), comme le coton Bt (incorporant des gènes de la bactérie Bacillus thuringiensis pour la résistance à certains ravageurs); Vognan et Fok (2019) montrent que, au Burkina Faso, seul pays qui a utilisé le coton Bt à une large échelle en Afrique l'Ouest avant de l'abandonner en 2016, les premiers impacts de cette nouvelle technologie dépendaient de nombreux facteurs et que les résultats attendus n'étaient pas forcément au rendez-vous, en particulier si la diversité des situations et des stratégies des producteurs n'était pas prise en compte. En outre, le recours aux OGM reste controversé compte tenu des risques, avérés ou non (perte de biodiversité, développement de résistances, coût pour les producteurs, etc.), notamment dans le cadre d'une transition agro-écologique, et est incompatible avec une labellisation biologique de la production. Une autre voie possible est l'écimage du cotonnier; au Mali, l'analyse de la perception des producteurs sur la technique manuelle de l'écimage du cotonnier (Diarra et al. 2020) montre que cette technique est facile à réaliser, diminue la chute des fleurs et des capsules, donne des plants plus courts s'étalant davantage et réduit l'utilisation et les coûts des pesticides ; mais elle augmente le travail et les coûts de maind'œuvre.

D'autres articles de ce numéro thématique abordent la durabilité des systèmes de production à base de coton dans une approche globale, à l'échelle des exploitations agricoles et de leur territoire. Ainsi, en zone cotonnière du Mali, sur la période 1987-2013, l'analyse des dynamiques agricoles, de la sécurité alimentaire et de l'accès aux services de santé et d'éducation dans un contexte de forte croissance démographique, montre une augmentation nette et progressive de la quantité de céréales produite par habitant, et des progrès similaires en matière d'accès aux infrastructures de santé et d'éducation (Soumaré et al., 2020). Un meilleur accès aux services et la diversification des activités agricoles en zone cotonnière sont des éléments mis en avant pour expliquer l'amélioration de la durabilité des exploitations agricoles dans cette zone. L'analyse des crédits de trésorerie des producteurs de coton du Bénin montre cependant que, vu les coûts de production du coton et l'insuffisance de crédits publics, le recours aux opérateurs informels de crédit à des taux d'intérêts élevés enferme les producteurs dans une trappe d'endettement et de pauvreté (Sossou et Fok, 2019). L'analyse du développement de l'anacarde en Côte d'Ivoire met en évidence que celui-ci est complémentaire à la culture du coton, tout en étant moins pénible, dégageant une meilleure marge brute et participant à la transformation du paysage agricole (Koffi et Oura, 2019); compte tenu de la forte demande mondiale en anacarde, et à la bonne adaptation de cette culture aux conditions des savanes cotonnières, cette diversification pourrait permettre de réduire la dépendance des économies locales au coton, tout en recélant elle aussi des risques importants, puisque cette production est également soumise aux aléas des cours des marchés mondiaux. 


\section{Conclusion}

Depuis plusieurs décennies, le secteur cotonnier s'est bien développé en AOC et a surmonté les crises, mais il est confronté aujourd'hui à de nouveaux défis. La culture du coton, qualifié «d'or blanc», a été un élément structurant des économies agricoles, et le moteur du développement rural de plusieurs pays producteurs. Cependant, la durabilité des systèmes de culture à base de cotonnier est souvent mise en cause, au vu de leurs impacts sur l'environnement et la santé humaine, liés à la lutte chimique contre les ravageurs du coton. Par contre, les avis des auteurs sont divergents quant aux impacts de ces systèmes sur la gestion de la fertilité des sols. Pour surmonter la crise des années 2000, tous les pays producteurs ont mis en place des réformes drastiques qui leur ont permis de faire face aux principales difficultés rencontrées, montrant ainsi la résilience du secteur cotonnier. Néanmoins, ce secteur et l'agriculture dans son ensemble sont confrontés à des défis importants pour lesquels la recherche doit se mobiliser. Dans le secteur cotonnier, les défis économiques portent sur la volatilité des prix sur le marché international, l'amélioration et la sécurisation des revenus des producteurs et du bien-être des communautés rurales, et sur les interférences politiques dans la gestion de la filière coton. Dans l'agriculture en général, y compris le secteur cotonnier, les défis sociaux portent sur l'inclusion des couches vulnérables de la population dans la dynamique de développement agricole (particulièrement les jeunes et les femmes) et le maintien des jeunes dans les exploitations. Les défis environnementaux portent sur le développement de pratiques agricoles plus écologiques (lutte contre la dégradation des sols, réduction de l'usage des pesticides, culture biologique, etc.) et adaptées aux nouvelles contraintes que pourrait imposer le changement climatique. Les défis sécuritaires concernent une partie des zones cotonnières de pays comme le Cameroun, le Nigéria et le Burkina Faso, où l'accès aux champs et l'évacuation de la production sont problématiques par endroit, ce qui renvoie au rôle des États en milieu rural.

\section{Références}

Afcot (Association française cotonnière). 2019. Production de coton en Afrique. https://afcot.org/actualites/production-de-coton-enafrique/.

Bachelier B, Balarabé O, Boulakia S, Demenois J, Gérardeaux E, Goebel R et al. 2018. Which ways forward for sustainable cotton production in Africa in the context of climate change? In : 77th Plenary Meeting of the International Cotton Advisory Committee (ICAC), 2-6 December 2018, Abidjan, Côte d'Ivoire. agritrop. cirad.fr/589934/.

Barry B. 2014. Le royaume du Waalo - Le Sénégal avant la conquête. Paris : Ed. Karthala, Coll. Hommes et Sociétés, 420 p.

Bassett TJ. 2002. Le coton des paysans, une révolution agricole (Côte d'Ivoire 1980-1999). Paris: IRD éditions, 292 p. DOI: $10.4000 /$ books.irdeditions. 10192.

Brévault T, Badiane D, Goebel R, Renou A, Tereta I, Clouvel P. 2019. Repenser la gestion des ravageurs du cotonnier en Afrique de 1'Ouest. Cahiers Agricultures 28(25): 8. DOI: 10.1051/cagri/ 2019024.
Castella JC, Deguine JP. 2006. Cycles phytosanitaires et viabilité des systèmes cotonniers. Cahiers Agricultures 15(1): 102-108. cahiers-agricultures/article/view/30567.

Commodafrica. 2015. Nouvelle hausse anticipée de la production de coton en Afrique de l'Ouest. http://www.commodafrica.com/ 14-09-2015-nouvelle-hausse-anticipee-de-la-production-de-cotonen-afrique-de-louest.

Commodafrica. 2019. Au nord du Burkina Faso, l'insécurité fait chuter de $70 \%$ la production agricole. http://www.commodafrica. com/29-10-2019-au-nord-du-burkina-faso-linsecurite-fait-chuterde-70-la-production-agricole.

Devèze JC, des Fontaines HD. 2005. Le devenir des agricultures familiales des zones cotonnières africaines: une mutation à conduire avec tous les acteurs. À partir des cas du Bénin, du Burkina Faso, du Cameroun et du Mali. Paris (France) : AFD EVA/ STR, 85 p.

Diarra M, Havard M, Soumaré M. 2020. La perception des avantages et contraintes de l'écimage du cotonnier par les producteurs du Mali. Cahiers Agricultures 29(10): 7. DOI: 10.1051/cagri/ 2020007.

Djagani KK, Fok M. 2019. Dangers potentiels de l'utilisation des insecticides dans la culture cotonnière au Togo de 1990 à 2010. Cahiers Agricultures 28(23): 9. DOI: 10.1051/cagri/2019023.

Dumont R. 1962. L'Afrique noire est mal partie. Paris (France) : Editions du Seuil, $251 \mathrm{p}$.

FAO. 2015. Measuring Sustainability in Cotton Farming Systems. Towards a Guidance Framework. Report. Rome (Italie): FAO, ICAC, 149 p. http://www.fao.org/3/a-i4170e.pdf.

FMI (Fonds monétaire international). 2014. Burkina Faso, Rapport $14 / 230,35 \mathrm{p}$.

Folefack DP, Ntsou Bakwowi J, Kpade PC. 2014. La crise de la filière cotonnière et sécurité alimentaire au Nord Cameroun. Journal of Applied Biosciences 75: 6221-6231. DOI: 10.4314/jab.v75i1.8.

Gafsi M, Mbétid-Bessane E. 2003. Stratégies des exploitations cotonnières et libéralisation de la filière. Cahiers Agricultures 12 (4): 253-260. cahiers-agricultures/article/view/30398.

Guichard L, Dedieu F, Jeufffroy MH, Meynard JM, Reau R, Sayini I. 2017. Le plan Ecophyto de réduction d'usage des pesticides en France : décryptage d'un échec et raisons d'espérer. Cahiers Agricultures 26(1): 14002. DOI: 10.1051/cagri/2017004.

Hauchart V. 2008. Culture du coton, pluviosité et dégradation des sols dans le Mouhoun (Burkina Faso). Sciences et changements planétaires/Sécheresse 19(2): 95-102.

ICAC. 2019. World cotton statistics, December 2019. www.icac.org (accès limité).

Intergovernmental panel on climate change (IPCC). 2014. Fifth Assessment Report-Climate Change. https://www.ipcc.ch/report/ ar5/syr/.

International Trade Centre (ITC). 2011. Women in cotton: Results of a Global Survey. Geneva: ITC. Xii +23 p. (Technical paper) Doc. No. SC-11-208.E. http://www.intracen.org/uploadedFiles/intrace norg/Content/Exporters/Sectors/Food_and_agri_business/Cotton/ AssetPDF/Women\%20in\%20cotton $\% 20-\overline{0} \% 209 \% 2011 \% 2011 \%$ 20FINAL.pdf.

Koffi YS, Oura KR. 2019. Les facteurs de l'adoption de l'anacarde dans le bassin cotonnier de Côte d'Ivoire. Cahiers Agricultures 28 (24): 8. DOI: 10.1051/cagri/2019025.

Lankoandé GD, Maradan D, Snon K, Thiombiano T, Zine K. 2011. Analyse économique du secteur du coton. Liens pauvreté et environnement. Rapport Final. Genève: Sba Ecosys.

Le Bars M, Sidibé F, Mandart E, Fabre J, Le Grusse P, Diakité CH. 2020. Evaluation des risques potentiels liés à l'utilisation de 
pesticides en culture cotonnière au Mali. Cahiers Agricultures 29 (4): 9. DOI: 10.1051/cagri/2020005.

Le Point Afrique. 2019. Mali: comment l'insécurité affecte le "grenier à riz» du pays. https://www.lepoint.fr/afrique/malicomment-1-insecurite-affecte-le-grenier-a-riz-du-pays24-07-2019-2326615 3826.php.

Levrat R. 2008. Le coton en Afrique Occidentale et Centrale avant 1950. Un exemple de la politique coloniale de la France. Paris (France): Editions l'Harmattan.

Mazoyer M, Roudard L. 2000. Histoire des agricultures du monde, du néolithique à la crise contemporaine. Paris (France) : Editions du Seuil, 705 p. https://www.seuil.com/ouvrage/histoire-des-agricultu res-du-monde-du-neolithique-a-la-crise-contemporaine-marcelmazoyer/9782020323970.

Mbétid-Bessane E, Havard M, Kadekoy-Tigagué D, Djondang K. 2010. Impacts des changements de politique dans un contexte de crise mondialisée sur les acteurs des filières cotonnières d'Afrique Centrale. Cahiers Agricultures 19(1): 21-27. DOI: 10.1684/ agr.2009.0339.

Michel B, Togola M, Téréta I, Traoré Navigué N. 2000. La lutte contre les ravageurs du cotonnier au Mali : problématique et évolution récente. Cahiers Agricultures 9(2): 109-115. cahiers-agricultures/ article/view/30228.

Nubukpo K. 2011. L'économie politique de la réforme des filières cotonnières d'Afrique de l'Ouest et du Centre : vers la convergence des modes d'organisation? Mondes en Développement 155(3): 93 109. DOI: $10.3917 / \mathrm{med} .155 .0093$.

Nubukpo K, Keita MS. 2006. Prix mondiaux, prix au producteur et avenir de la filière coton au Mali. Cahiers Agricultures 15(1): 3541. cahiers-agricultures/article/view/30557.

Orsenna E. 2006. Voyages aux pays du coton, petit précis de mondialisation. Paris : Fayard, $291 \mathrm{p}$.

Ouédraogo A, Gentil D. 2008. La microfinance en Afrique de l'Ouest. Histoires et innovations. Paris : Khartala Editions, $308 \mathrm{p}$.

Peltre-Wurtz J, Steck B. 1991. Les charrues de la Bagoué. Gestion paysanne d'une opération cotonnière en Côte d'Ivoire. Paris : IRD, 305 p. DOI: $10.4000 /$ books.irdeditions. 15566 .

Pichot J, Sédogo MP, Deguine JP. 2006. De nouveaux défis pour la recherche cotonnière dans un contexte difficile. Cahiers Agricultures 15(1): 150-157. cahiers-agricultures/article/view/30574.

Planetoscope. 2018. La production mondiale de coton. https://www. planetoscope.com/agriculture-alimentation/1178-production-mon diale-de-coton.html.

PR-PICA. 2018. Bulletin d'information du PR-PICA, numéro 20 Novembre-décembre. Programme Régional de production intégrée $\mathrm{du}$ coton en Afrique. http://prpica.org/IMG/pdf/bulletin_d_in fos_pr-pica_no20_ok.pdf.

Renaudin C. 2010 . Les riches heures et l'avenir incertain de la culture cotonnière en Afrique de l'Ouest et du Centre. EchoGeo 14. DOI: 10.4000/echogeo. 11955 .

RFI. 2015. Après une récolte de coton record, le Burkina réduit ses cultures OGM. www.rfi.fr/fr/emission/20150512-apres-unerecolte-coton-record-le-burkina-reduit-cultures-ogm.

Schneider K, Anderson CL, Gugerty MK. 2010. Gender \& Cash Cropping: Cotton in Sub-Saharan Africa. Washington: University of Washington, Evans School of Public Affairs, 11 p. https://evans. uw.edu/sites/default/files/Evans\%20UW Request $\% 2055$ Cotton $\% 20 \% 26 \% 20$ Gender_18\%20January\%202010.pdf.

Sossou KB, Fok M. 2019. Crédit de trésorerie des producteurs de coton au centre du Bénin: modalités et conséquences. Cahiers Agricultures 28(14): 9. DOI: 10.1051/cagri/2019015.

Soumaré M. 2008. Dynamiques et durabilité des systèmes agraires à base de coton au Mali. Thèse de doctorat unique, Université de Paris X Nanterre, 372 p. agritrop.cirad.fr/548140/.

Soumaré M, Traoré S, Havard M. 2020. Croissance démographique, sécurité alimentaire et accès à la santé et à l'éducation en zone cotonnière du Mali. Cahiers Agricultures 29: 2020036. DOI: 10.1051/cagri/2020036.

Tschirley D, Poulton C, Labaste P. 2009a. Organisation et performances des filières cotonnières africaines: leçons des réformes (Français). Washington, DC: Banque Mondiale, $223 \mathrm{p}$. http://documents.banquemondiale.org/curated/fr/ 355761468003294963/pdf/477210PUB0REPL1ch0Edition1Fi nal1PDF.pdf.

Tschirley D, Colin P, Gergely N, Labaste P. Baffes J. Boughton D, et al. 2009b. Méthode d'analyse des effets différentiels des réformes sur les filières cotonnières en Afrique. Cahiers Agricultures 18(5): 385-392. DOI: 10.1684/agr.2009.0334.

Vognan G, Fok M. 2019. Performance différentiée du coton Bt en début de diffusion: cas du Burkina Faso. Cahiers Agricultures 28 (26): 10. DOI: 10.1051/cagri/2019026.

Westerberg V, Golay A, Houndekon V, Costa L. 2017. L'économie de la dégradation de terre, le cas de la commune de Banikoara. Le coton est-il vraiment l'or blanc à Banikoara? GIZ, Economics of Land Degradation (ELD). www.eld-initiative.org.

Zoundi Sibiri J, Hussein K, Hitamana L. 2006. Libéralisation de la filière coton et innovation agricole en Afrique de l'Ouest. Cahiers Agricultures 15(1): 17-21. cahiers-agricultures/article/view/ 30555.

Citation de l'article : Soumaré M, Havard M, Bachelier B. 2020. Le coton en Afrique de l'Ouest et du Centre : de la révolution agricole à la transition agro-écologique. Cah. Agric. 29: 37. 